Теорія Ймовір. та Матем. Статист. Вип. 80, 2009
Theor. Probability and Math. Statist. No. 80, 2010, Pages 15-23 S 0094-9000(2010)00791-5

Article electronically published on August 18, 2010

\title{
LÉVY APPROXIMATION OF AN IMPULSE RECURRENT PROCESS WITH MARKOV SWITCHING
}

UDC 519.21

\author{
V. S. KOROLYUK, N. LIMNIOS, AND I. V. SAMOILENKO
}

\begin{abstract}
The weak convergence of an impulse recurrent process with Markov switching is proved in this paper for the scheme of the Lévy approximation. A modified Liptser semimartingale method is applied to the proof of the relative compactness of the process under consideration. The modification of the Liptser method used in the paper relies upon a solution of a singular perturbation problem instead of an ergodic theorem as used in the original Liptser method.
\end{abstract}

\section{INTRODUCTION}

The Lévy approximation is an interesting field for research in several theoretical and applied directions. Since Lévy processes are a standard object nowadays, the Lévy approximation is useful for the analysis of compound systems (see, for example, [1, 8]). The Lévy processes often appear in various applications such as risk theory, finance, queueing theory, physics, etc. Necessary definitions and results concerning the Lévy processes can be found, for example, in [1, 3, 8.

An impulse process represented by partial sums in a scheme of series

$$
\xi^{\varepsilon}(t)=\xi_{0}^{\varepsilon}+\sum_{k=1}^{\nu(t)} \alpha_{k}^{\varepsilon}\left(x_{k-1}^{\varepsilon}\right), \quad t \geq 0,
$$

is studied in [5], where the random variables $\alpha_{k}^{\varepsilon}(x), k \geq 1$, are independent and perturbed by a jump Markov process $x(t), t \geq 0$.

We consider a generalization of problem (1), namely

$$
\xi^{\varepsilon}(t)=\xi_{0}^{\varepsilon}+\sum_{k=1}^{\nu(t)} \alpha_{k}^{\varepsilon}\left(\xi_{k-1}^{\varepsilon}, x_{k-1}^{\varepsilon}\right), \quad t \geq 0 .
$$

Here the random variables $\alpha_{k}^{\varepsilon}(u, x), k \geq 1$, depend on the process $\xi^{\varepsilon}(t)$.

The convergence of the processes (2) is proved by combining two methods. Namely, we apply the Liptser method [6] based on the theory of semimartingales and solve a singular perturbation problem instead of applying an ergodic theorem as suggested by the original Liptser method. Our approach thus consists of two steps.

2000 Mathematics Subject Classification. Primary 60J55, 60B10, 60F17, 60K10; Secondary 60G46, $60 \mathrm{G} 60$.

Key words and phrases. Lévy approximation, semimartingale, Markov process, impulse recurrent process, piecewise deterministic Markov process, weak convergence, singular perturbation.

The authors are grateful to the University of Bielefeld for the hospitality and financial support in the framework of the project DFG 436 UKR 113/80/04-07. 
The first step is to prove the relative compactness for the semimartingale representation of the family $\xi^{\varepsilon}, \varepsilon>0$, with the help of the following two relations proposed in 7]:

$$
\lim _{c \rightarrow \infty} \sup _{\varepsilon \leq \varepsilon_{0}} \mathrm{P}\left\{\sup _{t \leq T}\left|\xi^{\varepsilon}(t)\right|>c\right\}=0
$$

(the compact containment condition) and

$$
\mathrm{E}\left|\xi^{\varepsilon}(t)-\xi^{\varepsilon}(s)\right|^{2} \leq k|t-s|
$$

for some positive constant $k$.

The second step is to prove the convergence of two components of the Markov process $\xi^{\varepsilon}(t), æ_{t}^{\varepsilon}:=æ\left(t / \varepsilon^{2}\right)$ with the help of the singular perturbation technique developed in [5]. Then we apply a theorem of [5].

The paper is organized as follows. In Section 22, we describe the impulse process (2) with scaled time and the Markov process with switching. The main results on the Lévy approximation are also given in Section 2. The proof of the main result is given in Section 3 .

\section{MAin RESUlt}

Consider the space $\mathbb{R}^{d}$ equipped with the norm $|\cdot|, d \geq 1$. Let $(E, \mathcal{E})$ be a standard phase space (that is, $E$ is a Polish space, while $\mathcal{E}$ is its Borel $\sigma$-algebra). We denote by $v^{*}$ and $c^{*}$ the transposition of a vector $v \in \mathbb{R}^{d}$ and a matrix $c \in \mathbb{R}^{d \times d}$, respectively. Let $C_{3}\left(\mathbb{R}^{d}\right)$ be the measure-determining class of real-valued bounded functions such that $g(u) /|u|^{2} \rightarrow 0$ as $|u| \rightarrow 0$ for $g \in C_{3}\left(\mathbb{R}^{d}\right)$ (see [4, 5] ).

Consider a family of random sequences $\alpha_{k}^{\varepsilon}(x), k=1,2, \ldots, x \in E$, where $E$ is a nonempty set indexed with a small parameter $\varepsilon>0$. For every $\varepsilon>0$ and every sequence $z_{k}, k \geq 0$, of elements of $\mathbb{R}^{d} \times E$, we assume that the random variables $\alpha_{k}^{\varepsilon}\left(z_{k-1}\right)$, $k \geq 1$, are independent. By $G_{u, x}^{\varepsilon}$ we denote the distribution function of $\alpha_{k}^{\varepsilon}(x)$, that is,

$$
G_{u, x}^{\varepsilon}(d v):=\mathrm{P}\left(\alpha_{k}^{\varepsilon}(u, x) \in d v\right), \quad k \geq 0, \varepsilon>0, x \in E, u \in \mathbb{R}^{d} .
$$

A Markov process $x(t), t \geq 0$, with switching on a standard phase space $(E, \mathcal{E})$ is defined by the generator

$$
\mathbf{Q} \varphi(x)=q(x) \int_{E} P(x, d y)[\varphi(y)-\varphi(x)],
$$

where $q(x), x \in E$, is the intensity function of jumps $x(t), t \geq 0$, while $P(x, d y)$ is the transient kernel of the embedded Markov chain $x_{n}, n \geq 0$, defined by $x_{n}=x\left(\tau_{n}\right), n \geq 0$, where $0=\tau_{0} \leq \tau_{1} \leq \cdots \leq \tau_{n} \leq \cdots$ are the jumps of the process $x(t), t \geq 0$. The corresponding jump counting process is defined by $\nu(t):=\max \left\{k \geq 0: \tau_{k} \leq t\right\}$. We impose a natural assumption on the counting process $\nu(t)$, namely

$$
\int_{0}^{t} \mathrm{E}[\varphi(s) d \nu(s)]<l_{1} \int_{0}^{t} \mathrm{E}(\varphi(s)) d s
$$

for all nonnegative increasing $\varphi(s)$ and $l_{1}>0$.

Now we define the family of jump Markov processes $x^{\varepsilon}(t):=x\left(t / \varepsilon^{2}\right), t \geq 0$, with an embedded Markov renewal process $x_{k}^{\varepsilon}, \tau_{k}^{\varepsilon}, k \geq 0$, and jump counting processes

$$
\nu^{\varepsilon}(t)=\nu\left(t / \varepsilon^{2}\right), \quad t \geq 0 \text {. }
$$

Thus $\tau_{k}^{\varepsilon}, k \geq 0$, are the moments when the process jumps, $x_{k}^{\varepsilon}:=x^{\varepsilon}\left(\tau_{k}^{\varepsilon}\right)$, and

$$
\nu^{\varepsilon}(t):=\max \left\{k \geq 0: \tau_{k}^{\varepsilon} \leq t\right\}
$$


The impulse processes $\xi^{\varepsilon}(t), t \geq 0, \varepsilon>0$, are defined on $\mathbb{R}^{d}$ in the scheme of series with a small parameter $\varepsilon \rightarrow 0, \varepsilon>0$, by the following sum (see [5, Section 9.2.1]):

$$
\xi^{\varepsilon}(t)=\xi_{0}^{\varepsilon}+\sum_{k=1}^{\nu\left(t / \varepsilon^{2}\right)} \alpha_{k}^{\varepsilon}\left(\xi_{k-1}^{\varepsilon}, x_{k-1}^{\varepsilon}\right), \quad t \geq 0 .
$$

Here

$$
\xi_{n}^{\varepsilon}:=\xi\left(\varepsilon^{2} \tau_{n}\right)=\xi_{0}^{\varepsilon}+\sum_{k=1}^{n} \alpha_{k}^{\varepsilon}\left(\xi_{k-1}^{\varepsilon}, x_{k-1}^{\varepsilon}\right) .
$$

It is worthwhile mentioning that the pair of processes $\xi^{\varepsilon}(t), x^{\varepsilon}(t), t \geq 0$, is an additive Markov process (see, for example, [5, Section 2.5]).

The Lévy approximation of the Markov impulse process (5) is considered under the following assumptions.

C1: The uniform ergodicity: The Markov process $x(t), t \geq 0$, is uniformly ergodic and $\pi(B), B \in \mathcal{E}$, is its stationary distribution.

C2: The Lévy approximation: The family of impulse processes $\xi^{\varepsilon}(t), t \geq 0$, satisfies the Lévy approximation conditions [5, Section 7.2.3].

L1: The initial condition:

$$
\sup _{\varepsilon>0} \mathrm{E}\left|\xi_{0}^{\varepsilon}\right| \leq C<\infty .
$$

L2: The approximation of the mean value:

$$
a^{\varepsilon}(u ; x)=\int_{\mathbb{R}^{d}} v G_{u, x}^{\varepsilon}(d v)=\varepsilon a_{1}(u ; x)+\varepsilon^{2}\left[a(u ; x)+\theta_{a}^{\varepsilon}(u ; x)\right]
$$

and

$$
c^{\varepsilon}(u ; x)=\int_{\mathbb{R}^{d}} v v^{*} G_{u, x}^{\varepsilon}(d v)=\varepsilon^{2}\left[c(u ; x)+\theta_{c}^{\varepsilon}(u ; x)\right],
$$

where the functions $a_{1}, a$, and $c$ are bounded.

L3: The Poisson approximation for the intensity kernel (see [4]):

$$
G_{g}^{\varepsilon}(u ; x)=\int_{\mathbb{R}^{d}} g(v) G_{u, x}^{\varepsilon}(d v)=\varepsilon^{2}\left[G_{g}(u ; x)+\theta_{g}^{\varepsilon}(u ; x)\right]
$$

for all $g \in C_{3}\left(\mathbb{R}^{d}\right)$, where the kernel $G_{g}(u ; x)$ is bounded for all $g \in C_{3}\left(\mathbb{R}^{d}\right)$, that is,

$$
\left|G_{g}(u ; x)\right| \leq G_{g}
$$

The constant $G_{g}$ depends on $g$. Here

$$
G_{g}(u ; x)=\int_{\mathbb{R}^{d}} g(v) G_{u, x}(d v), \quad g \in C_{3}\left(\mathbb{R}^{d}\right) .
$$

The terms $\theta_{a}^{\varepsilon}, \theta_{c}^{\varepsilon}$, and $\theta_{g}^{\varepsilon}$ are such that

$$
\sup _{x \in E}\left|\theta^{\varepsilon} .(u ; x)\right| \rightarrow 0, \quad \varepsilon \rightarrow 0 .
$$

L4: The balance condition:

$$
\int_{E} \rho(d x) a_{1}(u ; x)=0
$$

We also use the following assumptions. 
C3: The uniform square integrability:

$$
\lim _{c \rightarrow \infty} \sup _{x \in E} \int_{|v|>c} v v^{*} G_{u, x}(d v)=0,
$$

where the kernel $G_{u, x}(d v)$ is defined by relation (6) on the measure-determining class $C_{3}\left(\mathbb{R}^{d}\right)$.

C4: The linear growth: there exists a positive constant $L$ such that

$$
\begin{aligned}
|a(u ; x)| & \leq L(1+|u|), \quad|c(u ; x)| \leq L\left(1+|u|^{2}\right), \\
& \text { and }\left|G_{u, x}(v)\right| \leq L f(v)(1+|u|)
\end{aligned}
$$

for an arbitrary real-valued nonnegative function $f(x), x \in \mathbb{R}^{d}$, for which

$$
\int_{\mathbb{R}^{d} \backslash\{0\}}(1+f(x))|x|^{2} d x<\infty .
$$

The following is the main result of this paper.

Theorem 1. If assumptions $\mathbf{C 1 - C 4}$ hold, then we have the weak convergence

$$
\xi^{\varepsilon}(t) \Rightarrow \xi^{0}(t), \quad \varepsilon \rightarrow 0 .
$$

The limit process $\xi^{0}(t), t \geq 0$, is the Lévy process defined by its generator as follows:

$$
\mathbf{L} \varphi(u)=\left(\widehat{a}(u)-\widehat{a}_{0}(u)\right) \varphi^{\prime}(u)+\frac{1}{2} \sigma^{2}(u) \varphi^{\prime \prime}(u)+\lambda(u) \int_{\mathbb{R}^{d}}[\varphi(u+v)-\varphi(u)] G_{u}^{0}(d v)
$$

for $\sigma^{2}(u) \geq 0$, where

$$
\begin{gathered}
\widehat{a}(u)=q \int_{E} \rho(d x) a(u ; x), \quad \widehat{a}_{0}(u)=\int_{E} v G_{u}(d v), \quad G_{u}(d v)=q \int_{E} \rho(d x) G_{u, x}(d v), \\
\sigma^{2}(u)=2 \int_{E} \pi(d x)\left[\widetilde{a}_{1}(u ; x) R_{0} \widetilde{a}_{1}^{*}(u ; x)\right], \quad \widetilde{a}_{1}(u ; x):=q(x) \int_{E} P(x, d y) a_{1}(u ; x), \\
\lambda(u)=q G_{u}\left(\mathbb{R}^{d}\right), \text { and } G_{u}^{0}(d v)=G_{u}(d v) / G_{u}\left(\mathbb{R}^{d}\right) .
\end{gathered}
$$

\section{Proof of Theorem 1}

The proof of Theorem 1 is based on the semimartingale representation of impulse processes (5) and consists of two steps.

Step 1. We prove the relative compactness of the family of processes $\xi^{\varepsilon}(t), t \geq 0$, $\varepsilon>0$, by using the approach proposed in [6]. Recall that the space of all probability measures defined on a standard space $(E, \mathcal{E})$ is also a Polish space. Therefore the relative compactness and the density are equivalent.

We need the following auxiliary result.

Lemma 1. If condition $\mathbf{C 4}$ holds, then there exists a constant $k>0$ that does not depend on $\varepsilon$ but does depend on $T$ and such that

$$
\sup _{t \leq T}\left|\xi^{\varepsilon}(t)\right|^{2} \leq k_{T} .
$$

Corollary 1. If condition $\mathbf{C 4}$ holds, then the following compact embedding property is satisfied:

$$
\lim _{c \rightarrow \infty} \sup _{\varepsilon \leq \varepsilon_{0}} \mathrm{P}\left\{\sup _{t \leq T}\left|\xi^{\varepsilon}(t)\right|>c\right\}=0 .
$$

Proof. The proof of this corollary follows from Kolmogorov's inequality. 
Proof of Lemma 1 (by using the method of [6]). The impulse process (5) possesses the following semimartingale representation:

$$
\xi^{\varepsilon}(t)=u+B_{t}^{\varepsilon}+M_{t}^{\varepsilon},
$$

where $u=\xi_{0}^{\varepsilon}, B_{t}^{\varepsilon}$ is the predictable drift,

$$
B_{t}^{\varepsilon}=\sum_{k=1}^{\nu\left(t / \varepsilon^{2}\right)} a^{\varepsilon}\left(\xi_{k-1}^{\varepsilon}, x_{k-1}^{\varepsilon}\right)=A_{1}^{\varepsilon}(t)+A^{\varepsilon}(t)+\theta_{a}^{\varepsilon}(t),
$$

and where

$$
A_{1}^{\varepsilon}(t):=\varepsilon \sum_{k=1}^{\nu\left(t / \varepsilon^{2}\right)} a_{1}\left(\xi_{k-1}^{\varepsilon}, x_{k-1}^{\varepsilon}\right), \quad A^{\varepsilon}(t):=\varepsilon^{2} \sum_{k=1}^{\nu\left(t / \varepsilon^{2}\right)} a\left(\xi_{k-1}^{\varepsilon}, x_{k-1}^{\varepsilon}\right),
$$

$$
\left\langle M^{\varepsilon}\right\rangle_{t}=\varepsilon^{2} \sum_{k=1}^{\nu\left(t / \varepsilon^{2}\right)} c\left(\xi_{k-1}^{\varepsilon} ; x_{k-1}^{\varepsilon}\right)+\varepsilon^{2} \sum_{k=1}^{\nu\left(t / \varepsilon^{2}\right)} \int_{\mathbb{R}^{d} \backslash\{0\}} v v^{*} G\left(\xi_{k-1}^{\varepsilon}, d v ; x_{k-1}^{\varepsilon}\right)+\theta_{c}^{\varepsilon}(t) .
$$

Note that

$$
\sup _{0 \leq t \leq T}\left|\theta_{.}^{\varepsilon}(t)\right| \rightarrow 0, \quad \varepsilon \rightarrow 0,
$$

for all finite $T>0$.

When checking the compactness of the process $\xi^{\varepsilon}(t)$ we consider its two parts separately. The first part,

$$
A_{1}^{\varepsilon}(t)=\varepsilon \sum_{k=1}^{\nu\left(t / \varepsilon^{2}\right)} a_{1}\left(\xi_{k-1}^{\varepsilon} ; x_{k-1}^{\varepsilon}\right)
$$

being of order $\varepsilon$, can be represented in terms of the martingale

$$
\widetilde{\mu}_{t}^{\varepsilon}=\varphi^{\varepsilon}\left(A_{1}^{\varepsilon}(t)\right)+\varphi^{\varepsilon}\left(A_{1}^{\varepsilon}(0)\right)-\int_{0}^{t} \mathbf{L}^{\varepsilon} \varphi^{\varepsilon}\left(A_{1}^{\varepsilon}(s)\right) d s .
$$

Thus (see, for example, Theorem 1.2 in [5]) its quadratic characteristic is

$$
\left\langle\widetilde{\mu}^{\varepsilon}\right\rangle_{t}=\int_{0}^{t}\left[\mathbf{L}^{\varepsilon}\left(\varphi^{\varepsilon}\left(A_{1}^{\varepsilon}(s)\right)\right)^{2}-2 \varphi^{\varepsilon}\left(A_{1}^{\varepsilon}(s)\right) \mathbf{L}^{\varepsilon} \varphi^{\varepsilon}\left(A_{1}^{\varepsilon}(s)\right)\right] d s .
$$

Applying the operator $\mathbf{L}^{\varepsilon}=\varepsilon^{-2} \mathbf{Q}+\varepsilon^{-1} \mathbf{A}_{1}$ to the test function $\varphi^{\varepsilon}=\varphi+\varepsilon \varphi_{1}$ (here $\left.\mathbf{A}_{1}(u ; x) \varphi(v)=a_{1}(u ; x) \varphi^{\prime}(v)\right)$ we find the integrand of the form

$$
Q \varphi_{1}^{2}-2 \varphi_{1} Q \varphi_{1}
$$

This integrand does not depend on $\varepsilon$ and is bounded. Since the quadratic characteristic is bounded, $\widetilde{\mu}_{t}^{\varepsilon}$ is compact. Thus $\varphi\left(A_{1}^{\varepsilon}(t)\right)$ is also compact and uniformly bounded by the number $\varepsilon$.

Now we consider the other part of the process $\xi^{\varepsilon}(t)$, which has order $\varepsilon^{2}$.

Starting with $y(t), t \geq 0$, we define the process $y^{\dagger}(t)=\sup _{s \leq t}|y(s)|$. Then we derive from representation (8) that

$$
\left(\left(\xi^{\varepsilon}(t)\right)^{\dagger}\right)^{2} \leq 4\left[u^{2}+\left(\left(A^{\varepsilon}(t)\right)^{\dagger}\right)^{2}+\left(\left(M_{t}^{\varepsilon}\right)^{\dagger}\right)^{2}\right] .
$$

Now we use a result of Section 2.3 in [5], namely

$$
\sum_{k=1}^{\nu(t)} a\left(\xi_{k-1}^{\varepsilon}, x_{k-1}^{\varepsilon}\right)=\int_{0}^{t} a\left(\xi^{\varepsilon}(s), x^{\varepsilon}(s)\right) d \nu(s) .
$$


Condition C4 implies that, for sufficiently large $\varepsilon$,

$$
\left(A^{\varepsilon}(t)\right)^{\dagger}=\varepsilon^{2} \int_{0}^{t / \varepsilon^{2}} a\left(\xi^{\varepsilon}(s), x^{\varepsilon}(s)\right) d \nu(s) \leq L \varepsilon^{2} \int_{0}^{t / \varepsilon^{2}}\left(1+\left(\xi^{\varepsilon}(s)\right)^{\dagger}\right) d \nu(s) .
$$

According to Doob's inequality (see, for example, [7, Theorem 1.9.2]) we have

$$
\mathrm{E}\left(\left(M_{t}^{\varepsilon}\right)^{\dagger}\right)^{2} \leq 4\left|\mathrm{E}\left\langle M^{\varepsilon}\right\rangle_{t}\right| \text {. }
$$

Thus equality (9) and condition $\mathbf{C} 4$ imply that

$$
\begin{aligned}
\left|\left\langle M^{\varepsilon}\right\rangle_{t}\right| & =\left|\varepsilon^{2} \int_{0}^{t / \varepsilon^{2}} c\left(\xi^{\varepsilon}(s) ; x^{\varepsilon}(s)\right)+\varepsilon^{2} \int_{0}^{t / \varepsilon^{2}} \int_{\mathbb{R}^{d} \backslash\{0\}} v v^{*} G\left(\xi^{\varepsilon}(s), d v ; x_{s}^{\varepsilon}\right) d \nu(s)\right| \\
& \leq 2 L\left(1+r_{1}\right) \varepsilon^{2} \int_{0}^{t / \varepsilon^{2}}\left[1+\left(\left(\xi^{\varepsilon}(s)\right)^{\dagger}\right)^{2}\right] d \nu(s)
\end{aligned}
$$

where $r_{1}=\int_{\mathbb{R}^{d} \backslash\{0\}}|x|^{2} f(x) d x$.

Inequalities (10)-(12) together with condition (4) and the Cauchy-Bunyakovskiur inequality

$$
\left[\int_{0}^{t} \varphi(s) d s\right]^{2} \leq t \int_{0}^{t} \varphi^{2}(s) d s
$$

imply that

$$
\begin{aligned}
\mathrm{E}\left(\left(\xi^{\varepsilon}(t)\right)^{\dagger}\right)^{2} & \leq k_{1}+k_{2} \varepsilon^{2} \int_{0}^{t / \varepsilon^{2}} \mathrm{E}\left[\left(\left(\xi^{\varepsilon}(s)\right)^{\dagger}\right)^{2} d \nu(s)\right] \\
& \leq k_{1}+k_{2} l_{1} \varepsilon^{2} \int_{0}^{t / \varepsilon^{2}} \mathrm{E}\left(\left(\xi^{\varepsilon}(s)\right)^{\dagger}\right)^{2} d s=k_{1}+k_{2} l_{1} \int_{0}^{t} \mathrm{E}\left(\left(\xi^{\varepsilon}(s)\right)^{\dagger}\right)^{2} d s,
\end{aligned}
$$

where $k_{1}, k_{2}$, and $l_{1}$ are positive constants that do not depend on $\varepsilon$.

Now we apply the Gronwall inequality (see, for example, [2, p. 498]) and obtain

$$
\mathrm{E}\left(\left(\xi^{\varepsilon}(t)\right)^{\dagger}\right)^{2} \leq k_{1} \exp \left(k_{2} l_{1} t\right) .
$$

Therefore both parts of $\xi^{\varepsilon}(t)$ are compact and bounded, whence

$$
\sup _{t \leq T}\left|\xi^{\varepsilon}(t)\right|^{2} \leq k_{T}
$$

The lemma is proved.

Lemma 2. Let condition $\mathbf{C 4}$ hold. Then there exists a constant $k>0$ that does not depend on $\varepsilon$ and such that

$$
\mathrm{E}\left|\xi^{\varepsilon}(t)-\xi^{\varepsilon}(s)\right|^{2} \leq k|t-s| .
$$

Proof. Similarly to (10) we get

$$
\left|\xi^{\varepsilon}(t)-\xi^{\varepsilon}(s)\right|^{2} \leq 2\left|B_{t}^{\varepsilon}-B_{s}^{\varepsilon}\right|^{2}+2\left|M_{t}^{\varepsilon}-M_{s}^{\varepsilon}\right|^{2} .
$$

From Doob's inequality we derive

$$
\mathrm{E}\left|\xi^{\varepsilon}(t)-\xi^{\varepsilon}(s)\right|^{2} \leq 2 \mathrm{E}\left\{\left|B_{t}^{\varepsilon}-B_{s}^{\varepsilon}\right|^{2}+8\left|\left\langle M^{\varepsilon}\right\rangle_{t}-\left\langle M^{\varepsilon}\right\rangle_{s}\right|\right\} .
$$

Now (12) together with (4) and condition $\mathbf{C} 4$ implies that

$$
\left|B_{t}^{\varepsilon}-B_{s}^{\varepsilon}\right|^{2}+8\left|\left\langle M^{\varepsilon}\right\rangle_{t}-\left\langle M^{\varepsilon}\right\rangle_{s}\right| \leq k_{3}\left[1+\left(\left(\xi^{\varepsilon}(T)\right)^{\dagger}\right)^{2}\right]|t-s|,
$$

where $k_{3}$ is a positive constant that does not depend on $\varepsilon$.

The latter inequality and Lemma 1 prove Lemma 2 . 
The hypotheses of Corollary 1 and Lemma 2 are necessary and sufficient for the compactness of the family of processes $\xi^{\varepsilon}(t), t \geq 0, \varepsilon>0$.

Step 2. Now we consider the singular perturbation problem for the generator of the process $\xi^{\varepsilon}(t)$. In doing so, we will apply the following result. Let $C_{0}^{2}\left(\mathbb{R}^{d} \times E\right)$ be the space of real-valued functions being defined on $\mathbb{R}^{d} \times E$, being twice continuously differentiable with respect to the first argument, and vanishing at infinity. Also let $C\left(\mathbb{R}^{d} \times E\right)$ be the space of real-valued, continuous, bounded functions defined on $\mathbb{R}^{d} \times E$.

Theorem 2 ([5, Theorem 6.3]). Let the following conditions hold for a family of Markov processes $\xi^{\varepsilon}(t), t \geq 0, \varepsilon>0$ :

CD1: there exists a family of test functions $\varphi^{\varepsilon}(u, x)$ defined on $C_{0}^{2}\left(\mathbb{R}^{d} \times E\right)$ and such that

$$
\lim _{\varepsilon \rightarrow 0} \varphi^{\varepsilon}(u, x)=\varphi(u) \quad \text { uniformly in } u \text { and } x .
$$

CD2: The convergence

$$
\lim _{\varepsilon \rightarrow 0} \mathbf{L}^{\varepsilon} \varphi^{\varepsilon}(u, x)=\mathbf{L} \varphi(u)
$$

holds uniformly in $u$ and $x$. The family of functions $\mathbf{L}^{\varepsilon} \varphi^{\varepsilon}, \varepsilon>0$, is uniformly bounded where $\mathbf{L} \varphi(u)$ and $\mathbf{L}^{\varepsilon} \varphi^{\varepsilon}$ belong to $C\left(\mathbb{R}^{d} \times E\right)$.

CD3: The quadratic characteristics of the martingales corresponding to the Markov processes $\xi^{\varepsilon}(t), x^{\varepsilon}(t), t \geq 0, \varepsilon>0$, admit the following representation:

$$
\left\langle\mu^{\varepsilon}\right\rangle_{t}=\int_{0}^{t} \zeta^{\varepsilon}(s) d s
$$

where the random functions $\zeta^{\varepsilon}, \varepsilon>0$, are such that

$$
\sup _{0 \leq s \leq T} \mathrm{E}\left|\zeta^{\varepsilon}(s)\right| \leq c<+\infty .
$$

CD4: The initial values for all members of the family $\xi^{\varepsilon}(t), t \geq 0, \varepsilon>0$, are the same and such that

$$
\sup _{\varepsilon>0} \mathrm{E}\left|\zeta^{\varepsilon}(0)\right| \leq C<+\infty
$$

Then

$$
\xi^{\varepsilon}(t) \Rightarrow \xi(t), \quad \varepsilon \rightarrow 0 .
$$

Consider the two-component Markov process $\xi^{\varepsilon}(t), x_{t}^{\varepsilon}, t \geq 0$, characterized by the martingale

$$
\mu_{t}^{\varepsilon}=\varphi\left(\xi^{\varepsilon}(t), x_{t}^{\varepsilon}\right)-\int_{0}^{t} \mathbf{L}^{\varepsilon} \varphi\left(\xi^{\varepsilon}(s), x_{t}^{\varepsilon}\right) d s,
$$

whose generator $\mathbf{L}^{\varepsilon}$ admits the representation [5, Lemma 9.1]

$$
\mathbf{L}^{\varepsilon} \varphi(u, x)=\varepsilon^{-2} q(x)\left[\int_{E} P(x, d y) \int_{\mathbb{R}^{d}} G_{u, y}^{\varepsilon}(d z) \varphi(u+z, y)-\varphi(u, x)\right] .
$$

Similarly to [5, Lemma 9.2] one can prove the following result.

Lemma 3. The main term of the asymptotic expansion of the generator (13) is given by

$$
\begin{aligned}
\mathbf{L}^{\varepsilon} \varphi(u, v, x)= & \varepsilon^{-2} \mathbf{Q} \varphi(\cdot, \cdot, x)+\varepsilon^{-1} \mathbf{Q}_{0} a_{1}(u ; x) \varphi_{u}^{\prime}(u, \cdot, \cdot) \\
& +\mathbf{Q}_{0}\left[a(u ; x)-a_{0}(u ; x)\right] \varphi_{u}^{\prime}(u, \cdot, \cdot)+\mathbf{Q}_{0} \mathbf{G}_{u, x} \varphi(u, \cdot, \cdot),
\end{aligned}
$$


where

$$
\begin{gathered}
\mathbf{Q}_{0} \varphi(x):=q(x) \int_{E} P(x, d y) \varphi(y), \\
\mathbf{G}_{u, x} \varphi(u):=\int_{\mathbb{R}^{d}}[\varphi(u+v)-\varphi(u)] G_{u, x}(d v), \\
a_{0}(u ; x)=\int_{E} v G_{u, x}(d v) .
\end{gathered}
$$

The proof of Lemma 3 is similar to that of Lemma 9.2 in [5].

A solution of the singular perturbation problem for the test functions

$$
\varphi^{\varepsilon}(u, x)=\varphi(u)+\varepsilon \varphi_{1}(u, x)+\varepsilon^{2} \varphi_{2}(u, x)
$$

having the form $\mathbf{L}^{\varepsilon} \varphi^{\varepsilon}=\mathbf{L} \varphi+\theta^{\varepsilon} \varphi$ is found in the same way as in Lemma 9.3 of [5]. Hence

$$
\mathbf{L}=\Pi\left[\mathbf{Q}_{0}\left(\mathbf{A}(x)+\mathbf{G}_{u, x}\right)+\mathbf{Q}_{0} \mathbf{A}_{1}(x) R_{0} \mathbf{Q}_{0} \mathbf{A}_{1}(x)\right] \Pi,
$$

where

$$
\mathbf{A}(x) \varphi(u):=\left[a(u ; x)-a_{0}(u ; x)\right] \varphi^{\prime}(u)
$$

and

$$
\mathbf{A}_{1}(x) \varphi(u):=a_{1}(u ; x) \varphi^{\prime}(u) .
$$

After simple algebra we obtain equality (7) from (14).

Now we are ready to apply Theorem 2

We see from (13) and (14) that the solution of the singular perturbation problem for $\mathbf{L}^{\varepsilon} \varphi^{\varepsilon}(u, v ; x)$ satisfies conditions CD1 and CD2. Condition CD3 implies that the quadratic characteristics of the martingale corresponding to the two-component Markov process are relatively compact.

This result can also be derived from relation $(\mathrm{CCC})$ in view of [4] (see Corollary 1 and Lemma 2). Therefore condition CD3 follows from Corollary 1 and Lemma 2. Since $\xi^{\varepsilon}(0)=\xi^{0}(0)$, condition CD4 holds, too. All the assumptions of Theorem 2 hold, and thus we obtain the weak convergence $\xi^{\varepsilon}(t) \rightarrow \xi^{0}(t)$.

Theorem 1 is proved.

\section{BIBLIOGRAPHY}

1. J. Bertoin, Lévy Processes, Cambridge Tracts in Mathematics, vol. 121, Cambridge University Press, Cambridge, 1996. MR1406564 (98e:60117)

2. S. N. Ethier and T. G. Kurtz, Markov Processes: Characterization and Convergence, Wiley, New York, 1986. MR838085 (88a:60130)

3. I. I. Gikhman and A. V. Skorokhod, Theory of Stochastic Processes, vol. 1, Nauka, Moscow, 1971; English. transl., Springer, Berlin, 1974. MR0341539 (49:6287)

4. J. Jacod and A. N. Shiryaev, Limit Theorems for Stochastic Processes, Springer-Verlag, Berlin, 1987. MR959133 (89k:60044)

5. V. S. Korolyuk and N. Limnios, Stochastic Systems in Merging Phase Space, World Scientific, Singapore, 2005. MR2205562(2007a:60004)

6. R. Sh. Liptser, The Bogolyubov averaging principle for semimartingales, Trudy Mat. Inst. Steklov., vol. 202, 1993, pp. 175-189; English transl. in Proc. Steklov Inst. Math., vol. 202, 1994, pp. 143-153. MR.1383305 (97c:60117) 
7. R. Sh. Liptser and A. N. Shiryayev, Theory of Martingales, Nauka, Moscow, 1986; English. transl., Kluwer Academic Publishers, Dordrecht, The Netherlands, 1989. MR1022664 (90j:60046)

8. K.-I. Sato, Lévy Processes and Infinitely Divisible Distributions, Cambridge Studies in Advanced Mathematics, vol. 68, Cambridge University Press, Cambridge, 1999. MR1739520 (2003b:60064)

Institute of Mathematics, National Academy of Sciences of Ukraine, Kiev, Ukraine

E-mail address: korol@imath.kiev.ua

Laboratoire de Mathématiques Appliquées, Université de Technologie de Compiègne, FRANCE

E-mail address: nlimnios@dma.utc.fr

Institute of Mathematics, National Academy of Sciences of Ukraine, Kiev, Ukraine

E-mail address: isamoil@imath.kiev.ua

Received 9/FEB/2009

Translated by S. V. KVASKO 